ORIGINAL RESEARCH

S. Inano

H. Takao

N. Hayashi

O. Abe

K. Ohtomo

\title{
Effects of Age and Gender on White Matter Integrity
}

BACKGROUND AND PURPOSE: DTI provides a sensitive measure of change in the microstructure of white matter integrity. The purpose of our study was to investigate age-related changes, sex differences, and age-by-sex interactions in white matter integrity (FA, AD, and RD) across the whole brain with a large sample.

MATERIALS AND METHODS: A total of 857 healthy subjects (mean age $=56.1 \pm 9.9$ years; age range $=$ 24.9-84.8 years) were included in this study. All subjects were scanned at 3T. With use of TBSS, we examined the effects of age and sex on FA, AD, and RD in the white matter.

RESULTS: Global FA was negatively correlated with age $\left(R^{2}=0.18, P<.0001\right)$, and global AD and RD were positively correlated with age (AD: $R^{2}=0.02, P<.0001$; RD: $\left.R^{2}=0.19, P<.0001\right)$. The correlation between age and global AD, however, was weak. Voxelwise analysis revealed a number of regions where FA was negatively correlated with age, with most of these regions showing a significant positive correlation between $\mathrm{RD}$ and age. There was a significant age-related FA increase in several white matter regions. Voxelwise analysis also revealed many regions where $F A, A D$, or $R D$ differed between men and women; however, no region showed a significant interaction between age and sex.

CONCLUSIONS: Our results suggest that age-related changes in white matter integrity are more strongly associated with myelin sheath degeneration than with axonal degeneration, and that, in some specific regions, the number of remyelinated axons might increase with age. Our results also suggest that there are no sex differences in the aging process of the white matter.

ABBREVIATIONS: $A D=$ axial diffusivity; ANCOVA = analysis of covariance; $A$ SSET = array spatial sensitivity encoding technique; BET = Brain Extraction Tool; FA = fractional anisotropy; FDT = FMRIB's Diffusion Toolbox; FNIRT = FMRIB's nonlinear registration tool; FSL = FMRIB Software Library; FWE = family-wise error; MD = mean diffusivity; MMSE = Mini-Mental State Examination; $\mathrm{MNI}=$ Montreal Neurological Institute; RD = radial diffusivity; TBSS = tract-based spatial statistics

D TI is an MR imaging technique that is sensitive to random thermal motions and provides a unique and sensitive probe for the architecture of biologic tissues. DTI has been widely used to study the integrity of the white matter in healthy brains and in a variety of neuropsychological diseases. FA and MD are the most widely used diffusion parameters. The most pervasive findings from DTI aging research are agerelated increases in $\mathrm{MD}$ and decreases in FA. ${ }^{1-10}$

Recently, DTI aging studies have examined eigenvalues. ${ }^{11}$ The first eigenvalue is referred to as axial diffusivity (diffusion parallel to the axon fibers), ${ }^{12,13}$ whereas the average of the second and third eigenvalues is termed radial diffusivity (diffusivity perpendicular to the axonal fibers). ${ }^{12,13}$ Animal studies have showed that lowered $\mathrm{AD}$ reflects axonal injury ${ }^{14,15}$ and that increases in $\mathrm{RD}$ are linked to incomplete myelination, ${ }^{13}$ drug-induced demyelination, ${ }^{15}$ and loss of myelin following axon injury. ${ }^{14,15}$ It is suggested that in the human brain, age-related increases in RD are due to demyelina-

Received February 11, 2011; accepted after revision March 28.

From the Departments of Radiology (S.I., H.T., K.O.) and Computational Diagnostic Radiology and Preventive Medicine (N.H.), Graduate School of Medicine, University of Tokyo, Tokyo, Japan; and Department of Radiology (O.A.), Nihon University School of Medicine, Tokyo, Japan.

Paper previously presented in part at: 16th Annual Meeting of the Organization for Human Brain Mapping, June 6-10, 2010; Barcelona, Spain.

Please address correspondence to Sachiko Inano, Department of Radiology, Graduate School of Medicine, University of Tokyo, 7-3-1 Hongo, Bunkyo-ku, Tokyo 113-8655, Japan; e-mail: inano-sim@umin.ac.jp

http://dx.doi.org/10.3174/ajnr.A2785 tion ${ }^{16-19}$ or a decrease in axonal packing attenuation, ${ }^{16,19}$ and age-related decreases in $\mathrm{AD}$ are caused by axonal degeneration and the subsequent gliosis that follows an ischemic incident or lesion formation. ${ }^{17,20,21}$

Previous DTI aging studies that included AD and RD measures revealed 3 prominent patterns of age differences in diffusivity. In the first pattern, age-related decreases in FA are primarily associated with a significant increase in RD but not $\mathrm{AD} .{ }^{3,16,22-24}$ In the second pattern, age-related decreases in FA are associated with significant increases in both $\mathrm{RD}$ and AD. ${ }^{2,16,22,25,26}$ In the third pattern, age-related decreases in FA are associated with significant increases in RD and decreases in AD. ${ }^{2,16,22}$ All 3 patterns are characterized by an increase in RD with aging. The results of these studies also suggest that agerelated microstructural alterations in white matter differ among regions.

Sex effects on global or regional white matter integrity still remain unclear. Some DTI studies showed no sex difference, ${ }^{5,6,27,28}$ whereas others showed a significant sex difference. ${ }^{4,25,29-32}$ In one DTI study that investigated sex effects on white matter integrity across the brain, significant sex differences in FA were seen in the right deep temporal region. ${ }^{4}$

Previous DTI studies have also investigated age-by-sex interaction in diffusion characteristics. ${ }^{4-6,25,32-35}$ Some studies reported that the interaction between age and sex was not significant, ${ }^{4,6,32}$ and some studies reported significant interactions of diffusion properties between age and sex..$^{25,33,34}$ Both sex differences and age-by-sex interactions of white matter integrity require further evaluation with a large sample size. 
The purpose of our study was to investigate age-related changes, sex differences, and age-by-sex interactions in white matter integrity (FA, $\mathrm{AD}$, and $\mathrm{RD}$ ) across the whole brain with a large sample. To the best of our knowledge, this sample is the largest among studies that have investigated age-related changes or sex differences in white matter integrity.

\section{Materials and Methods}

\section{Subjects}

A total of 857 healthy subjects ( 310 women and 547 men; mean age $=$ $56.1 \pm 9.9$ years; age range $=24.9-84.8$ years) were included in this study. All subjects were volunteers who underwent private health screening. None of the subjects had a history of neuropsychiatric disorder, including serious head trauma, psychiatric disorders, or alcohol/substance abuse or dependence. The MMSE score was $29.2 \pm$ 0.9 (range $=27-30)$. Two radiologists (with 6 and 10 years of experience, respectively) reviewed all scans (including T2-weighted images) and found no gross abnormalities such as infarct, hemorrhage, or brain tumors in any of the subjects. The Fazekas score (range, 0-3) was 0 (absence) or 1 (caps, pencil-thin lining, and/or punctuate foci). ${ }^{36}$ Subjects with grade $0(n=309$, mean age $=50.7 \pm 8.5$ years $)$ were significantly younger than subjects with grade $1(n=548$, mean age $=59.2 \pm 9.3$ years; Mann-Whitney test, $P<.0001)$. The ethical committee of our institute approved this study. After a complete explanation of the study to each subject, written informed consent was obtained.

\section{Imaging Data Acquisition}

MR data were obtained on two 3T Signa HDx scanners (GE Medical Systems, Milwaukee, Wisconsin) of the exact same model with an 8-channel brain phased-array coil. Diffusion tensor images were acquired by using a single-shot spin-echo echo-planar sequence in 50 axial sections $(\mathrm{TR}=13,200 \mathrm{~ms}$; $\mathrm{TE}=62 \mathrm{~ms} ; \mathrm{FOV}=288 \mathrm{~mm}$; section thickness $=3 \mathrm{~mm}$ with no gap; acquisition matrix $=96 \times 96$; $\mathrm{NEX}=$ 1 ; image matrix $=256 \times 256$ ). Diffusion weighting was applied along 13 noncollinear directions with a b-value of 1000 seconds $/ \mathrm{mm}^{2}$, and a single volume was collected with no diffusion gradients applied $\left(b_{0}\right)$. Parallel imaging (ASSET) was used with an acceleration factor of 2.0. The reconstructed voxel dimensions were $1.125 \times 1.125 \times 3.0 \mathrm{~mm}$.

\section{Image Processing}

Image analysis was performed by using TBSS $1.2,{ }^{37,38}$ which is part of FSL 4.1 (http://www.fmrib.ox.ac.uk/fsl). ${ }^{39}$ First, the raw diffusion data were corrected for eddy current distortion and head motion by using FDT 2.0, ${ }^{39}$ and corrected for spatial distortion due to gradient nonlinearity by using grad_unwarp. ${ }^{40}$ Following brain extraction by using BET 2.1, ${ }^{41} \mathrm{FA}, \mathrm{AD}$, and $\mathrm{RD}$ maps were created by fitting a tensor model to the diffusion data by using FDT. The FA data of all subjects were then aligned into MNI 152 space by using FNIRT 1.0, ${ }^{39}$ which uses a b-spline representation of the registration warp field. The FMRIB58_FA standard-space image was used as the target. Next, a mean FA image was created and thinned to create a mean FA skeleton, which represents the centers of all tracts common to the group. The mean FA skeleton image was thresholded at an FA value of 0.2 to prevent inclusion of nonskeleton voxels. ${ }^{37}$ The aligned FA data of each subject were then projected onto this skeleton. The $\mathrm{AD}$ and $\mathrm{RD}$ data were also aligned into MNI 152 space and projected onto the mean FA skeleton (by using the FA data to find the projection vectors).

\section{Statistical Analyses}

Differences in skeleton average $\mathrm{FA}, \mathrm{AD}$, and $\mathrm{RD}$ were tested by using ANCOVA, with skeleton average $\mathrm{FA}, \mathrm{AD}$, or $\mathrm{RD}$ as the dependent variable, and age, sex, age-by-sex, and scanner as independent variables. The relationship between age and skeleton average FA, AD, or $\mathrm{RD}$ was evaluated by using a simple linear regression analysis and a quadratic regression analysis. These statistical analyses were performed by using JMP 8.0 (SAS Institute, Cary, North Carolina). A $P$ value of $<.05$ was considered to indicate a statistically significant difference.

Voxelwise analyses of the skeletonized data were performed by using permutation-based, voxelwise nonparametric testing (as implemented in the Randomise Tool part of FSL) ${ }^{42}$ First, we identified areas in which $\mathrm{FA}, \mathrm{AD}$, or RD was significantly correlated with age. Sex and scanner were also included as covariates. Next, we identified areas with a significant effect of sex on FA, AD, or RD. Finally, we identified areas with a significant interaction between age and sex. Significance levels for $t$ tests (1-tailed) were set at $P<.025$, corrected for multiple comparisons by using the FWE rate (voxel-level inference). We computed $2 t$ contrasts (positive, negative) for $t$ tests. The number of permutations was 5000 .

\section{Results}

Age and Sex Effects on Global FA, AD, and RD

ANCOVA for skeleton average $\mathrm{FA}, \mathrm{AD}$, and $\mathrm{RD}$ revealed a main effect of age (FA, $F=170.4, P<.0001 ; \mathrm{AD}, F=19.5, P<$ $.0001 ; \mathrm{RD}, F=185.3, P<.0001)$. There was no sex-by-age interaction $(\mathrm{FA}, F=0.68, P=.41 ; \mathrm{AD}, F=0.51, P=.47 ; \mathrm{RD}$, $F=1.32, P=.25)$. Although there was a main effect of sex on $\mathrm{FA}$ and $\mathrm{AD}(\mathrm{FA}, F=43.1, P<.0001 ; \mathrm{AD}, F=28.4, P<.0001)$, there was no significant main effect of sex on RD $(F=3.10$, $P=.08)$.

Simple regression analysis showed a significant negative correlation between age and FA $\left(R^{2}=0.18, P<.0001\right.$, FA $=$ $0.46-0.00062 \times$ Age $[y])$. A significant positive correlation was seen between age and $\mathrm{AD}\left(R^{2}=0.02, P<.0001, \mathrm{AD}\right.$ $\left[\times 10^{-3} \mathrm{~mm}^{2} / \mathrm{s}\right]=1.12+0.00033 \times$ Age $\left.[\mathrm{y}]\right)$ and between age and $\mathrm{RD}\left(R^{2}=0.19, P<.0001, \mathrm{RD}\left[\times 10^{-3} \mathrm{~mm}^{2} / \mathrm{s}\right]=0.51+\right.$ $0.00098 \times$ Age $[y])$. Quadratic regression analysis also showed a significant relationship between age and FA $\left(R^{2}=0.18, P<\right.$ $.0001 ; \mathrm{FA}=0.46-0.00062 \times$ Age $[\mathrm{y}]-0.0000047 \times($ Age $[\mathrm{y}]$ $\left.-56.1)^{\wedge} 2\right)$, between age and $\mathrm{AD}\left(R^{2}=0.04, P<.0001 ; \mathrm{AD}\right.$ $\left[\times 10^{-3} \mathrm{~mm}^{2} / \mathrm{s}\right]=1.12+0.00029 \times$ Age $[\mathrm{y}]+0.000023 \times$ (Age $\left.[y]-56.1)^{\wedge} 2\right)$, and between age and $\operatorname{RD}\left(R^{2}=0.21, P<\right.$ $.0001 ; \mathrm{RD}\left[\times 10^{-3} \mathrm{~mm}^{2} / \mathrm{s}\right]=0.51+0.00095 \times$ Age $[\mathrm{y}]+$ $0.000020 \times($ Age $[y]-56.1) \wedge 2)$. The quadratic term was significant for $\operatorname{AD}(P<.0001)$ and $\operatorname{RD}(P=.0004)$ but not for FA $(P=.22)$. Fig 1 shows scatterplots of skeleton average FA, $\mathrm{AD}$, and $\mathrm{RD}$ versus age.

\section{Age Effects on Regional FA, $A D$, and RD}

Voxelwise analysis revealed a number of regions in which FA values were negatively correlated with age: the body of the corpus callosum, fornix, bilateral anterior limb of the internal capsule, bilateral external capsule, bilateral anterior cingulum, bilateral inferior longitudinal fasciculus, and cerebellum (Fig 2, Table). A significant age-related increase in FA was seen in the splenium of the corpus callosum, bilateral corona radiata, bilateral posterior limb of the internal capsule, and bilateral 

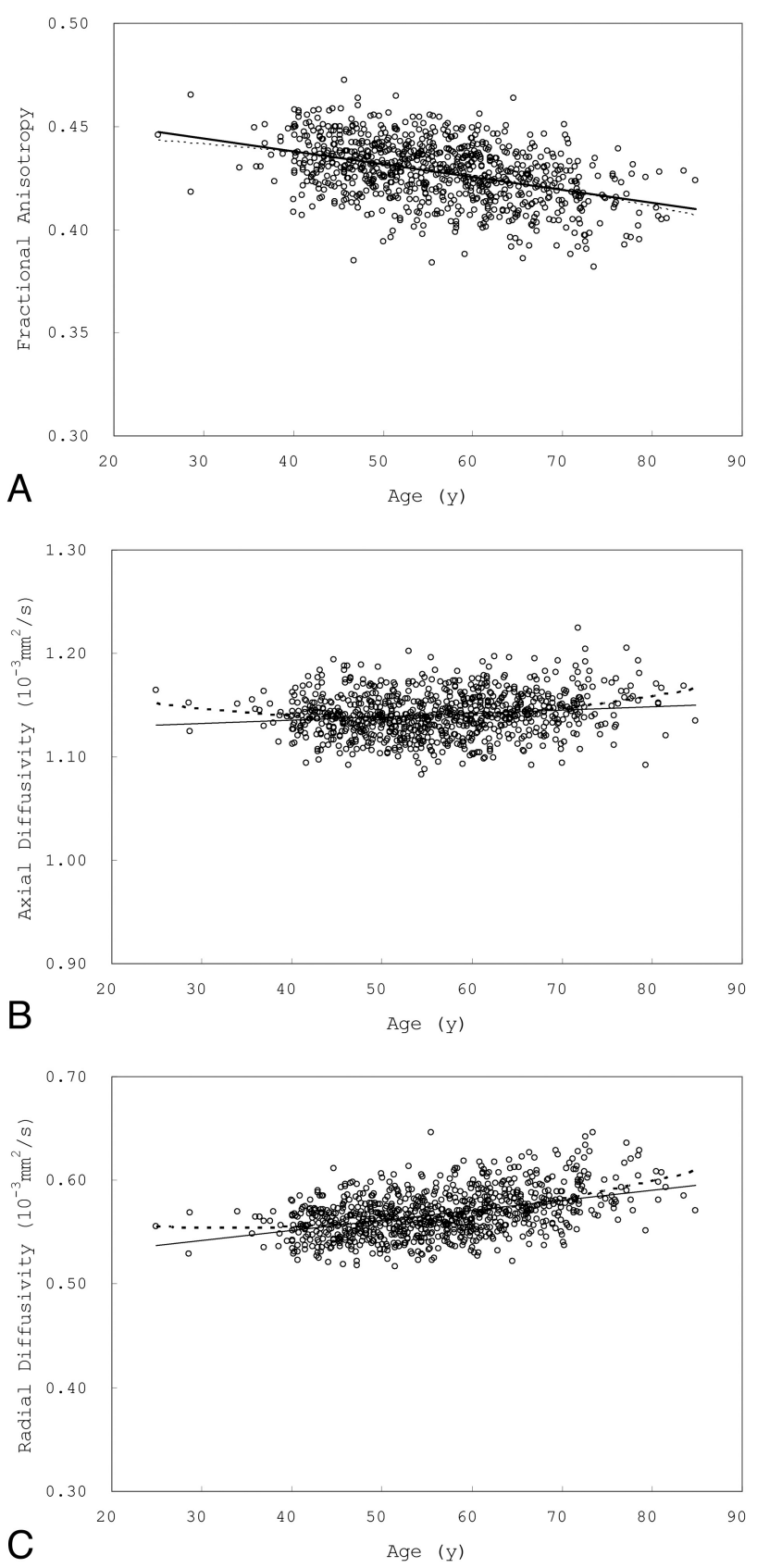

Fig 1. Effects of age on global $F A, A D$, and RD. $A$, Scatterplot of skeleton average $F A$ versus age; $B$, scatterplot of skeleton average $\mathrm{AD}$ versus age; $C$, scatterplot of skeleton average $\mathrm{RD}$ versus age. A significant negative correlation was seen between age and global FA. A significant positive correlation was seen between age and global $A D$ and between age and global $\mathrm{RD}$; however, the correlation between age and global $\mathrm{AD}$ was weak. Simple linear regression curves (solid lines) and quadratic regression curves (dotted lines) are shown. The quadratic term was significant in $A D$ and $R D$ but not in FA.

superior longitudinal fasciculus. A number of regions were identified in which $\mathrm{AD}$ or RD values were positively correlated with age (Fig 2, Table). Among the white matter regions showing a negative correlation between age and FA, a positive correlation between age and $\mathrm{AD}$ was seen in the body of the corpus callosum and fornix. Among the white matter regions showing a negative correlation between age and FA, a positive correlation between age and $\mathrm{RD}$ was seen in most regions. Among the white matter regions showing a positive correlation between age and FA, a positive correlation between age

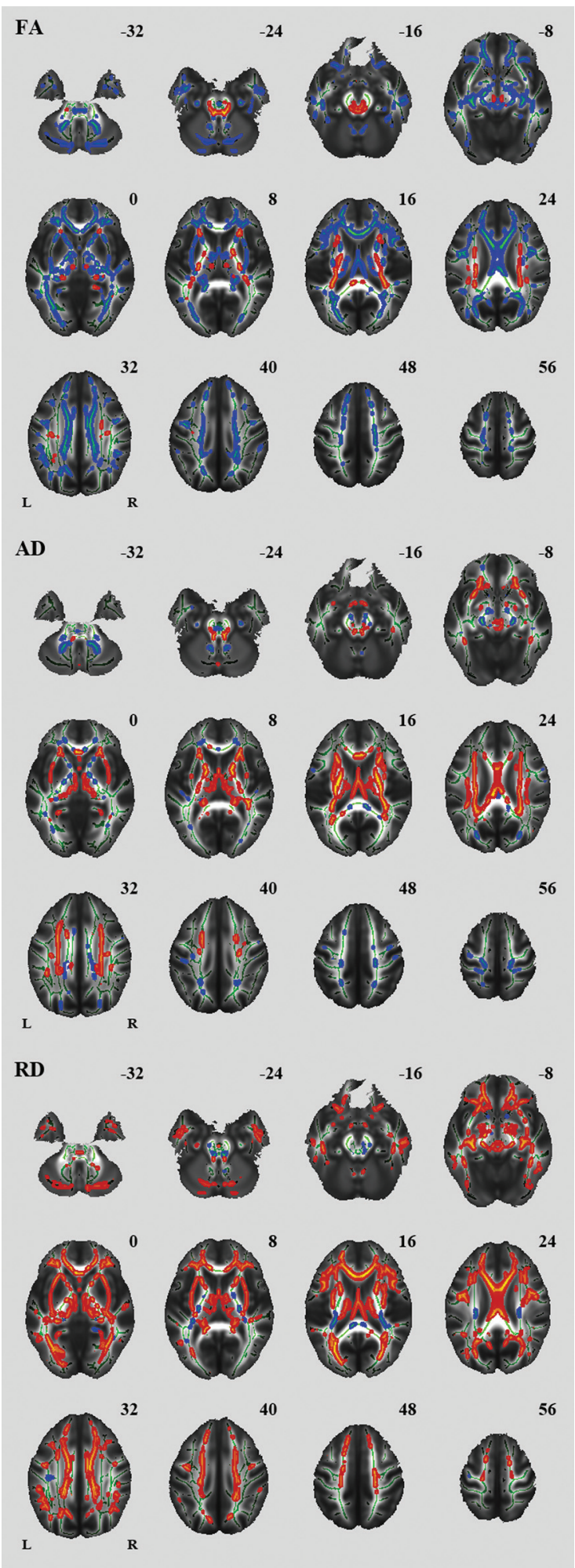

Fig 2. Effects of age on regional $F A, A D$, and $R D$. White matter tracts in red-yellow show a significant age-related increase in $F A, A D$, and $R D$. White matter tracts in blue-green show a significant age-related decrease in $F A, A D$, and $R D$.

and $\mathrm{AD}$ was seen in regions except for the splenium of the corpus callosum. Among the white matter regions showing a positive correlation between age and FA, a negative correlation between age and RD was seen in the splenium of the corpus callosum. 


\begin{tabular}{|c|c|}
\hline \multicolumn{2}{|c|}{$\begin{array}{l}\text { Summary of TBSS analysis of aging effects on white matter } \\
\text { integrity }\end{array}$} \\
\hline & Region \\
\hline \multicolumn{2}{|l|}{ FA } \\
\hline Age-related decrease & $\begin{array}{l}\text { Body of corpus callosum } \\
\text { Fornix }\end{array}$ \\
\hline & $\begin{array}{c}\text { Anterior limb of internal capsule } \\
\text { External capsule } \\
\text { Anterior cingulum } \\
\text { Inferior longitudinal fasciculus } \\
\text { Cerebellum }\end{array}$ \\
\hline Age-related increase & $\begin{array}{l}\text { Splenium of corpus callosum } \\
\text { Corona radiata } \\
\text { Posterior limb of internal capsule } \\
\text { Superior longitudinal fasciculus }\end{array}$ \\
\hline \multicolumn{2}{|l|}{$A D$} \\
\hline Age-related increase & $\begin{array}{c}\text { Body of corpus callosum } \\
\text { Fornix } \\
\text { Posterior limb of internal capsule } \\
\text { Corona radiata } \\
\text { Superior longitudinal fasciculus }\end{array}$ \\
\hline \multicolumn{2}{|r|}{ 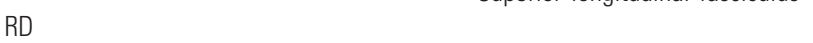 } \\
\hline $\begin{array}{l}\text { Age-related decrease } \\
\text { Age-related increase }\end{array}$ & $\begin{array}{c}\text { Splenium of corpus callosum } \\
\text { Body of corpus callosum } \\
\text { Fornix } \\
\text { Inferior longitudinal fasciculus } \\
\text { Anterior limb of internal capsule } \\
\text { External capsule } \\
\text { Cerebellum }\end{array}$ \\
\hline
\end{tabular}

Fig 3 shows scatterplots of mean $\mathrm{FA}, \mathrm{AD}$, and $\mathrm{RD}$ versus age for voxels that showed a significant positive correlation between age and FA. Fig 4 shows scatterplots of mean FA, AD, and $\mathrm{RD}$ versus age for voxels that showed a significant negative correlation between FA and age.

\section{Sex Effects on Regional FA, $A D$, and RD}

We also identified a number of regions in which FA, AD, or $\mathrm{RD}$ differed between women and men (Fig 5). Significantly higher FA was seen in men compared with women in many regions, including the splenium of the corpus callosum, bilateral corona radiata, posterior limb of the internal capsule, cerebral peduncle, external capsule, bilateral cingulum, bilateral superior longitudinal fasciculus, and bilateral middle cerebellar peduncle. Significantly higher FA was seen in women compared with men in the column of the fornix.

\section{Interaction Between Age and Sex}

The voxelwise analysis showed no regions with a significant interaction between age and sex, except for a few voxels.

\section{Discussion}

We found a significant negative correlation between age and global FA, as well as significant positive correlations between age and global $\mathrm{AD}$, and between age and global RD. The correlation between age and global $\mathrm{AD}$, however, was weak, which suggests that the previously reported age-related increase in global MD is due mainly to age-related increase in global RD. The voxelwise analysis revealed a number of regions in which FA was negatively correlated with age, with most of these regions showing a significant positive correlation between $\mathrm{RD}$ and age. The results of the voxelwise analysis
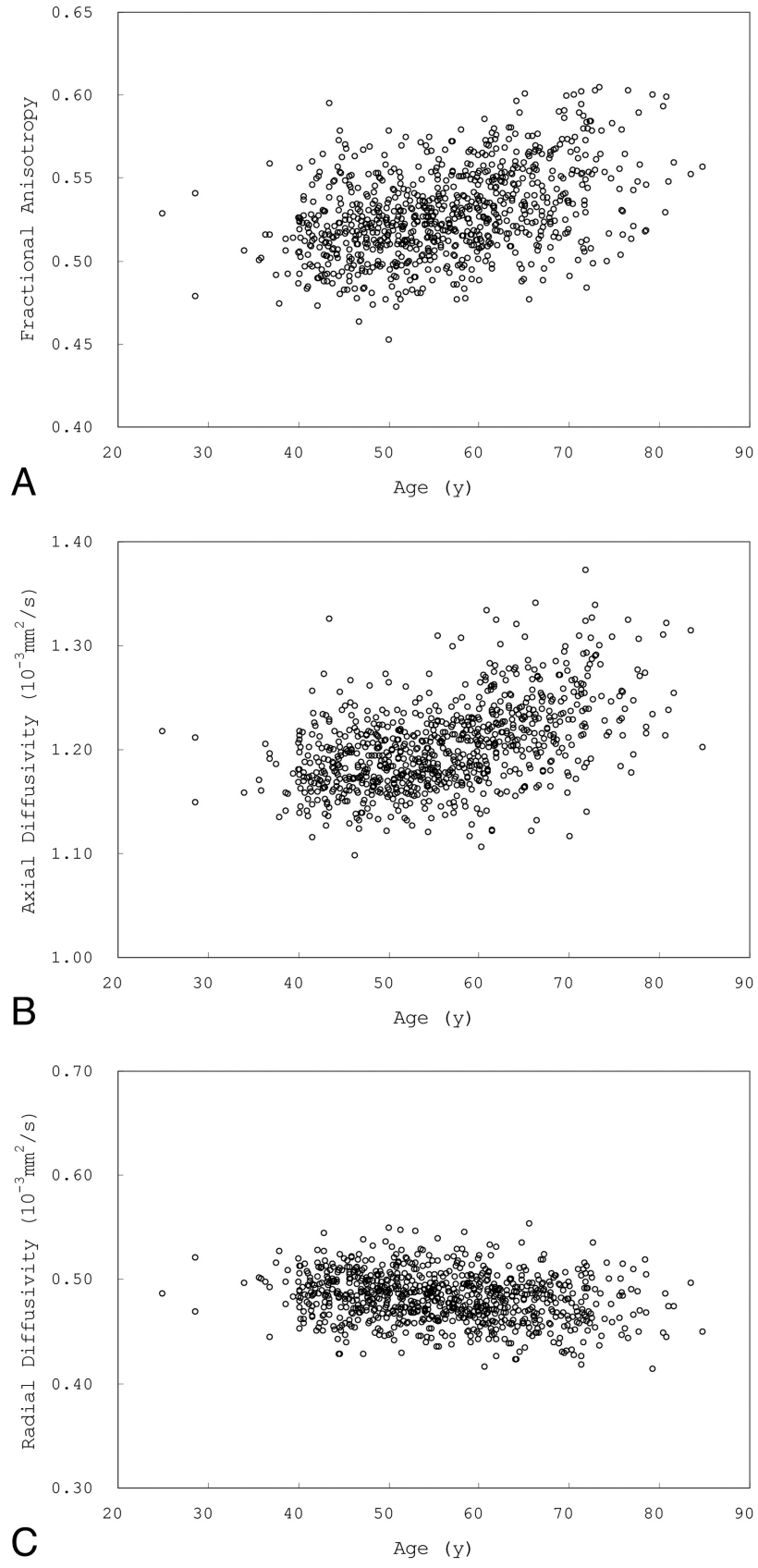

Fig 3. Scatterplots of mean $F A(A), A D(B)$, and $R D(C)$ versus age for voxels that showed a positive correlation between age and $F A$.

indicate that age-related decrease in FA is associated with agerelated increase in RD. In addition, the voxelwise analysis revealed several regions in which FA values were positively correlated with age. We identified a number of regions in which $\mathrm{FA}, \mathrm{AD}$, or RD differed between men and women; however, no region showed a significant interaction between age and sex.

To the best of our knowledge, the present sample size is the largest among studies that have investigated age-related changes or sex differences in white matter integrity. In addition, we used TBSS, a relatively new approach for analyzing differences in white matter, to circumvent the problem of cross-subject alignment and contamination due to differences in brain morphology. 

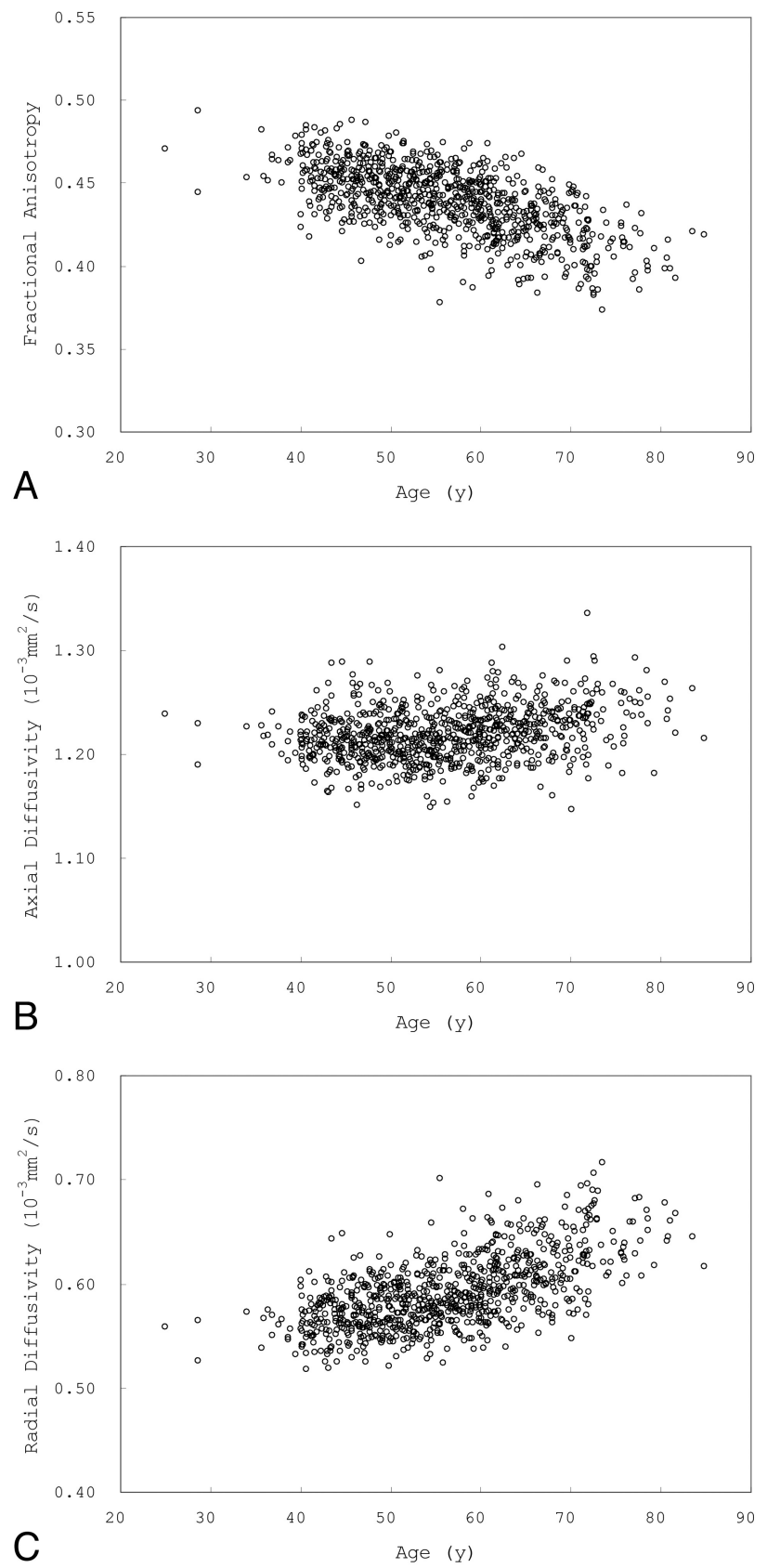

Fig 4. Scatterplots of mean $F A(A), A D(B)$, and $R D(C)$ versus age for voxels that showed a negative correlation between $F A$ and age

\section{Global Effects of Age on White Matter Integrity}

In agreement with the findings of previous studies, ${ }^{4,33,43}$ we found a significant negative correlation between age and global FA, as well as significant positive correlations between age and global $\mathrm{AD}$, and between age and global RD. The correlation between age and global $\mathrm{AD}$ was weak. The present regression analysis of global DTI indices demonstrated that the quadratic term was significant in $\mathrm{AD}$ and $\mathrm{RD}$ but not in FA. These results are consistent with recent DTI studies that used the voxel-based approach to investigate adult aging. ${ }^{33,43}$

\section{Regional Effects of Age on White Matter Integrity}

A notable finding of the present study is a significant agerelated increase in FA in several white matter regions. Several

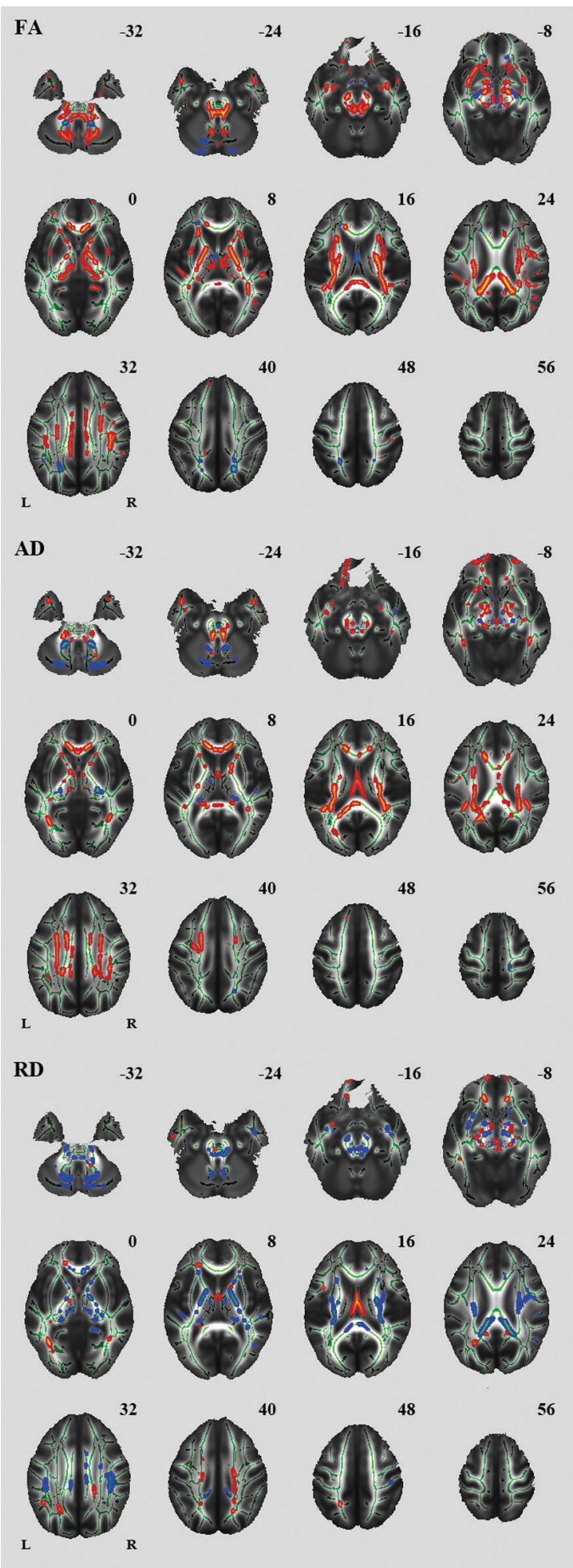

Fig 5. Effects of sex on regional $F A, A D$, and $R D$. White matter tracts in red-yellow show a significantly higher $F A, A D$, and $R D$ in men compared with women. White matter tracts in blue-green show a significantly higher $F A, A D$, and $R D$ in women compared with men.

previous studies reported an age-related increase in FA in some white matter regions such as the splenium of the corpus callosum $^{5,6,43,44}$; however, no study has reported an age-related increase in FA, as found in the present study. This finding may indicate that these regions are resistant to age-related degeneration. 
Similarly to previous reports, ${ }^{16,22-26,45}$ the present results showed that age-related decreases in FA are associated with 2 region-specific patterns of age differences in diffusivity measures: a pattern of radial increase only, and a pattern of both radial and axial increases. Previous DTI aging studies also reported that age-related decreases in FA were associated with radial increase and an axial decrease pattern ${ }^{16,22,45}$; however, we found this pattern only in a small part of the bilateral inferior longitudinal fasciculus.

Previous DTI reports have described varying behavior of $\mathrm{AD}$ with age. Age-related decreases in $\mathrm{AD}$ are thought to be caused by axonal degeneration and the subsequent gliosis. ${ }^{14,20-22}$ Age-related increases in AD were commonly reported in previous DTI aging studies. ${ }^{2,16,22,25,26}$ Although the cause of this pattern remains unclear, increase in $\mathrm{AD}$ is a relatively specific pattern in normal aging.

In animal studies, myelin breakdown has been associated with increased RD, while axonal degeneration is reflected by changes in $\mathrm{AD} .{ }^{14}$ In normal aging, some myelin sheaths degenerate as a consequence of their degenerating axons. Based on the findings of earlier studies of Wallerian nerve fiber degeneration, there is little doubt that the degeneration of an axon is inexorably followed by the breakdown and degeneration of its myelin sheath. ${ }^{46}$ In other cases, myelin sheaths degenerate even when the axon is intact. Such axons are remyelinated by a series of internodes that are much shorter than the original ones and are composed of thinner sheaths. Oligodendrocytes, myelin-forming cells of the central nervous system, remain active during aging. Rivers et $\mathrm{al}^{47}$ calculated that about $20 \%$ of all oligodendrocytes in the adult corpus callosum are generated during adulthood, and 5\% of adult-born oligodendrocytes in the cerebral cortex appear to be involved in elaboration of the myelin sheath. We consider that the present results reflect a part of the biologic mechanism mentioned above. In our analysis age-related increases in $\mathrm{RD}$ were observed in most white matter regions that showed a negative correlation between age and FA. This result would reflect agerelated increases in the degenerated myelin sheaths.

Some of the white matter regions showing a positive correlation between age and FA in the present study, especially those in the splenium of the corpus callosum that showed a negative correlation between age and $\mathrm{RD}$, might indicate regions where the number of remyelinated axons increased with age. Age-related decreases in RD may reflect age-related increases in the thinner sheaths of the remyelinated fibers. This may be supported by the results of a previous study, which found that more myelin-forming oligodendrocytes are found in the adult corpus callosum than in the cerebral cortex in mice. $^{48}$

\section{Effects of Sex on White Matter Integrity}

We found a significantly higher FA in men compared with women in many regions, including the splenium of the corpus callosum and bilateral cingulum, which is consistent with the findings of previous studies. ${ }^{29,31} \mathrm{Hsu}$ et $\mathrm{al}^{43}$ found significant sex differences in FA in the right deep temporal region and the left anterior limb of the internal capsule. In the present study, we found sex differences in a small part of the bilateral anterior limb of the internal capsule. Lebel et $\mathrm{al}^{30}$ reported that males had significantly higher FA in the superior frontal region of the corpus callosum; however, we found no sex difference in this region. We also found a significantly higher FA in men compared with women in other white matter regions, and significantly higher FA in women compared with men in the column of the fornix; however, previous studies that analyzed white matter microstructure in adults, ${ }^{4,25}$ and during adolescence or in children, ${ }^{48}$ reported no significant sex difference for regions in which we found significant sex differences in the present study.

\section{Interaction of Age and Sex}

We found no age-by-sex interaction in global or regional analyses of $\mathrm{FA}, \mathrm{AD}$, and $\mathrm{RD}$, suggesting the absence of sex difference in the aging process of white matter. Hsu et al reported no significant effect in age-by-sex interaction in a mean global analysis of DTI indices (2010), ${ }^{43}$ and none of the ROIs in the genu and splenium of the corpus callosum and 7 predefined regions in each hemisphere showed a significant age-by-sex interaction (2008). ${ }^{4}$ These results are consistent with the pres-

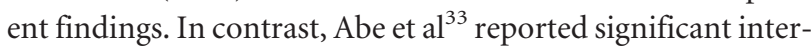
action between sex and age, suggesting that the rates of global FA decrease with age were greater for men than for women, and that in regional FA and $\mathrm{MD}$, men showed a steeper FA decline and accelerated MD increase against age in multiple brain regions. Kochunov et $\mathrm{al}^{34}$ reported significant age ${ }^{2}$ bysex interactions for average FA, cingulum, corticospinal tract, and splenium of the corpus callosum, and that males had slightly higher average FA values and slightly higher rates of FA decline.

\section{Limitations}

There are some limitations of our study. Methodological limitations include a relatively large voxel size and a relatively small number of the diffusion directions. However, the large sample size of our study would overcome these limitations. We used the diffusion tensor, the most widely applied mathematical model used to represent the angular variability of diffusion, for investigating the effects of age and sex on white matter integrity. An important limitation of the model is that it cannot accurately represent voxels containing multiple fiber bundles with different orientations. ${ }^{49}$ Its use is another limitation of our study.

\section{Conclusions}

Our study showed that age-related decrease in FA is more strongly associated with age-related increase in RD than with age-related change in $\mathrm{AD}$. These results suggest that age-related changes in white matter are more strongly associated with myelin sheath degeneration than with axonal degeneration. One of the new findings of the present study is that some white matter regions showed age-related increases in FA. We also found sex differences in white matter integrity in a number of regions. No regions showed a significant interaction between age and sex. These results suggest a lack of sex difference in the aging process of white matter and that sex differences in white matter integrity may arise during its maturational process. 


\section{Acknowledgments}

The authors thank Dr. Eriko Maeda and Dr. Takeharu Yoshikawa for their help in collecting data.

Disclosures: Kuni Ohtomo, Research Support (including provision of equipment or materials): Bayer/DaiichiSankyo; Details: Fund-Bayer $¥ 2000$ 000/year; DaiichiSankyo $¥ 2000000$ /year.

\section{References}

1. Abe $\mathrm{O}$, Aoki S, Hayashi $\mathrm{N}$, et al. Normal aging in the central nervous system: quantitative MR diffusion-tensor analysis. Neurobiol Aging 2002;23:433-41

2. Barrick TR, Charlton RA, Clark CA, et al. White matter structural decline in normal ageing: a prospective longitudinal study using tract-based spatial statistics. Neuroimage 2010;51:565-77

3. Giorgio A, Santelli L, Tomassini V, et al. Age-related changes in grey and white matter structure throughout adulthood. Neuroimage 2010;51:943-51

4. Hsu JL, Leemans A, Bai CH, et al. Gender differences and age-related white matter changes of the human brain: a diffusion tensor imaging study. Neuroimage 2008;39:566-77

5. Kennedy KM, Raz N. Aging white matter and cognition: differential effects of regional variations in diffusion properties on memory, executive functions, and speed. Neuropsychologia 2009;47:916-27

6. Kennedy KM, Raz N. Pattern of normal age-related regional differences in white matter microstructure is modified by vascular risk. Brain Res 2009;1297:41-56

7. Pfefferbaum A, Sullivan EV, Hedehus M, et al. Age-related decline in brain white matter anisotropy measured with spatially corrected echo-planar diffusion tensor imaging. Magn Reson Med 2000;44:259-68

8. Pfefferbaum A, Adalsteinsson E, Sullivan EV. Frontal circuitry degradation marks healthy adult aging: evidence from diffusion tensor imaging. Neuroimage 2005;26:891-99

9. Salat DH, Tuch DS, Greve DN, et al. Age-related alterations in white matter microstructure measured by diffusion tensor imaging. Neurobiol Aging 2005;26:1215-27

10. Yoon B, Shim YS, Lee KS, et al. Region-specific changes of cerebral white matter during normal aging: a diffusion-tensor analysis. Arch Gerontol Geriatr 2008;47:129-38

11. Pierpaoli C, Basser PJ. Toward a quantitative assessment of diffusion anisotropy. Magn Reson Med 1996;36:893-906

12. Basser PJ. Inferring microstructural features and the physiological state of tissues from diffusion-weighted images. NMR Biomed 1995;8:333-44

13. Song SK, Sun SW, Ramsbottom MJ, et al. Dysmyelination revealed through MRI as increased radial (but unchanged axial) diffusion of water. Neuroimage 2002;17:1429-36

14. Song SK, Sun SW, Ju WK, et al. Diffusion tensor imaging detects and differentiates axon and myelin degeneration in mouse optic nerve after retinal ischemia. Neuroimage 2003;20:1714-22

15. Song SK, Yoshino J, Le TQ, et al. Demyelination increases radial diffusivity in corpus callosum of mouse brain. Neuroimage 2005;26:132-40

16. Bennett IJ, Madden DJ, Vaidya CJ, et al. Age-related differences in multiple measures of white matter integrity: a diffusion tensor imaging study of healthy aging. Hum Brain Mapp 2010;31:378-90

17. Berman JI, Mukherjee P, Partridge SC, et al. Quantitative diffusion tensor MRI fiber tractography of sensorimotor white matter development in premature infants. Neuroimage 2005;27:862-71

18. Le Bihan D. Looking into the functional architecture of the brain with diffusion MRI. Nat Rev Neurosci 2003;4:469-80

19. Pierpaoli C, Jezzard P, Basser PJ, et al. Diffusion tensor MR imaging of the human brain. Radiology 1996;201:637-48

20. Pierpaoli C, Barnett A, Pajevic S, et al. Water diffusion changes in wallerian degeneration and their dependence on white matter architecture. Neuroimage 2001;13:1174-85

21. Thomalla G, Glauche V, Koch MA, et al. Diffusion tensor imaging detects early wallerian degeneration of the pyramidal tract after ischemic stroke. Neuroimage 2004;22:1767-74

22. Burzynska AZ, Preuschhof C, Bäckman L, et al. Age-related differences in white matter microstructure: region-specific patterns of diffusivity. Neuroimage 2010;49:2104-12

23. Davis SW, Dennis NA, Buchler NG, et al. Assessing the effects of age on long white matter tracts using diffusion tensor tractography. Neuroimage 2009;46:530-41
24. Zhang $\mathrm{Y}, \mathrm{Du}$ AT, Hayasaka S, et al. Patterns of age-related water diffusion changes in human brain by concordance and discordance analysis. Neurobiol Aging 2010;31:1991-2001

25. Sullivan EV, Rohlfing T, Pfefferbaum A. Quantitative fiber tracking of lateral and interhemispheric white matter systems in normal aging: relations to timed performance. Neurobiol Aging 2008;31:464-81

26. Vernooij MW, de Groot M, van der Lugt A, et al. White matter atrophy and lesion formation explain the loss of structural integrity of white matter in aging. Neuroimage 2008;43:470-77

27. Lee CE, Danielian LE, Thomasson D, et al. Normal regional fractional anisotropy and apparent diffusion coefficient of the brain measured on a $3 \mathrm{~T}$ MR scanner. Neuroradiology 2009;51:3-9

28. Ota M, Obata T, Akine Y, et al. Age-related degeneration of corpus callosum measured with diffusion tensor imaging. Neuroimage 2006;31:1445-52

29. Huster RJ, Westerhausen R, Kreuder F, et al. Hemispheric and gender related differences in the midcingulum bundle: a DTI study. Hum Brain Mapp 2009;30:383-91

30. Lebel C, Caverhill-Godkewitsch S, Beaulieu C. Age-related regional variations of the corpus callosum identified by diffusion tensor tractography. Neuroimage 2010;52:20-31

31. Oh JS, Song IC, Lee JS, et al. Tractography-guided statistics (TGIS) in diffusion tensor imaging for the detection of gender difference of fiber integrity in the midsagittal and parasagittal corpora callosa. Neuroimage 2007;36:606-16

32. Sullivan EV, Adalsteinsson E, Hedehus M, et al. Equivalent disruption of regional white matter microstructure in ageing healthy men and women. $\mathrm{Neu}$ roreport 2001;12:99-104

33. Abe $\mathrm{O}$, Yamasue $\mathrm{H}$, Yamada $\mathrm{H}$, et al. Sex dimorphism in gray/white matter volume and diffusion tensor during normal aging. NMR Biomed 2010;23:446-58

34. Kochunov P, Williamson DE, Lancaster J, et al. Fractional anisotropy of water diffusion in cerebral white matter across the lifespan. Neurobiol Aging 2010 Jan 30. [Epub ahead of print]

35. Schneiderman JS, Buchsbaum MS, Haznedar MM, et al. Diffusion tensor anisotropy in adolescents and adults. Neuropsychobiology 2007;55:96-111

36. Fazekas F, Chawluk JB, Alavi A, et al. MR signal abnormalities at $1.5 \mathrm{~T}$ in Alzheimer's dementia and normal aging. AJR Am J Roentgenol 1987;149: 351-56

37. Smith SM, Jenkinson M, Johansen-Berg H, et al. Tract-based spatial statistics: voxelwise analysis of multi-subject diffusion data. Neuroimage 2006;31:1487505

38. Smith SM, Johansen-Berg H, Jenkinson M, et al. Acquisition and voxelwise analysis of multi-subject diffusion data with tract-based spatial statistics. Nat Protoc 2007;2:499-503

39. Smith SM, Jenkinson M, Woolrich MW, et al. Advances in functional and structural MR image analysis and implementation as FSL. Neuroimage 2004; 23(suppl 1):S208-19

40. Jovicich J, Czanner S, Greve D, et al. Reliability in multi-site structural MRI studies: effects of gradient non-linearity correction on phantom and human data. Neuroimage 2006;30:436-43

41. Smith SM. Fast robust automated brain extraction. Hum Brain Mapp 2002;17:143-55

42. Nichols TE, Holmes AP. Nonparametric permutation tests for functional neuroimaging: a primer with examples. Hum Brain Mapp 2002;15:1-25

43. Hsu JL, Van Hecke W, Bai CH, et al. Microstructural white matter changes in normal aging: a diffusion tensor imaging study with higher-order polynomial regression models. Neuroimage 2010;49:32-43

44. Michielse S, Coupland N, Camicioli R, et al. Selective effects of aging on brain white matter microstructure: a diffusion tensor imaging tractography study. Neuroimage 2010;52:1190-201

45. Sala S, Agosta F, Pagani E, et al. Microstructural changes and atrophy in brain white matter tracts with aging. Neurobiol Aging 2010 Jun 29. [Epub ahead of print]

46. Guillery, R. W. Light- and electron-microscopical studies of normal and degenerating axons. In: Nauta WJH, Ebbesson SOE, eds. Contemporary Research Methods in Neuroanatomy. New York: Springer-Verlag; 1970:77-105

47. Rivers LE, Young KM, Rizzi M, et al. PDGFRA/NG2 glia generate myelinating oligodendrocytes and piriform projection neurons in adult mice. Nat NeurosC 2008;11:1392-401

48. Schmithorst VJ, Holland SK, Dardzinski BJ. Developmental differences in white matter architecture between boys and girls. Hum Brain Mapp 2008;29:696-710

49. Jbabdi S, Behrens TE, Smith SM. Crossing fibres in tract-based spatial statistics. Neuroimage 2010;49:249-56 